\title{
BMJ Open Efficacy of home-based non- pharmacological interventions for treating depression: a systematic review and network meta-analysis of randomised controlled trials
}

\author{
Kanokporn Sukhato, ${ }^{1}$ Manote Lotrakul, ${ }^{2}$ Alan Dellow, ${ }^{3}$ Pichai Ittasakul, ${ }^{2}$ \\ Ammarin Thakkinstian, ${ }^{4}$ Thunyarat Anothaisintawee ${ }^{1}$
}

To cite: Sukhato K, Lotrakul M, Dellow A, et al. Efficacy of home-based nonpharmacological interventions for treating depression: a systematic review and network meta-analysis of randomised controlled trials. BMJ Open 2017;7:e014499. doi:10.1136/ bmjopen-2016-014499

- Prepublication history and additional material are available. To view please visit the journal online (http://dx.doi.org/10. 1136/bmjopen-2016-014499).

Received 28 September 2016 Revised 17 May 2017 Accepted 25 May 2017

\section{(a) CrossMark}

${ }^{1}$ Department of Family Medicine, Faculty of Medicine, Ramathibodi Hospital, Mahidol University, Bangkok, Thailand ${ }^{2}$ Department of Psychiatry, Faculty of Medicine, Ramathibodi Hospital, Mahidol University, Bangkok, Thailand

${ }^{3}$ Faculty of Medicine,

Ramathibodi Hospital, Mahidol University, Bangkok, Thailand

${ }^{4}$ Section for Clinical

Epidemiology and Biostatistics, Faculty of Medicine,

Ramathibodi Hospital, Mahidol University, Bangkok, Thailand

Correspondence to

Dr Thunyarat Anothaisintawee; thunyarat.ano@mahidol.ac.th

\section{ABSTRACT}

Objectives To systematically review and compare the efficacy of all available home-based non-pharmacological treatments of depression.

Design Systematic review and network meta-analysis of randomised controlled trials.

Data sources Medline, Scopus and Cumulative Index to Nursing and Allied Health Literature (CINAHL) databases were searched since inceptions to 7 August 2016.

Eligibility criteria Randomised controlled trials comparing the efficacy of home-based nonpharmacological interventions with usual care of patients with depression were included in the review.

Main outcomes Depression symptom scores and disease remission rates at the end of treatment.

Results Seventeen studies were included in the review. Home-based non-pharmacological interventions were categorised as (1) home-based psychological intervention, (2) home-based exercise intervention, (3) combined homebased psychological intervention with exercise intervention and (4) complementary medicine. Complementary medicine approaches were excluded from the metaanalysis due to heterogeneity. The standardised mean differences of post-treatment depression symptom scores between usual care groups and home-based psychological intervention, home-based exercise intervention and combined home-based psychological intervention with exercise intervention were â’ 0.57 (95\% Cl â’ 0.84 to

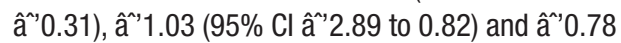
(95\% Cl â'1.09 to â' 0.47$)$, respectively. These results suggest that only home-based psychological intervention and combined home-based psychological intervention with exercise intervention could significantly decrease depression scores. Compared with usual care groups, the disease remission rate was also significantly higher for home-based psychological intervention (pooled risk ratio=1.53; 95\%â€\%ol 1.19 to 1.98) and combined home-based psychological intervention with exercise intervention (pooled risk ratio=3.47; 95\%â€\%ol 2.11 to 5.70). Of all the studied interventions, combined homebased psychological intervention with exercise intervention had the highest probability of resulting in disease remission.
Strengths and limitations of this study

- Our analysis provides the first comprehensive review of the efficacy of home-based non-pharmacological interventions in treating depression.

- A comprehensive search was undertaken to identify as many relevant studies as possible.

- We performed a network meta-analysis to compare the efficacy of home-based interventions in order to identify the best treatment regimen.

- The quality of included studies in the area of allocation concealment was not optimal.

- Participants in our included studies were aware of their own interventions and the outcomes were subjective.

Conclusion Our study confirms the efficacy of homebased psychological intervention and combined homebased psychological intervention with exercise intervention in the treatment of depression. Combined home-based psychological intervention and exercise intervention was the best treatment and should be considered for inclusion in clinical guidelines for managing depression.

\section{INTRODUCTION}

Recent studies have highlighted the international recognition of depression as one of the leading global burdens of disease. ${ }^{1}$ Depression is associated with greater morbidity and mortality and with increasing health service use and costs. ${ }^{2}$ In addition, untreated depression has been recognised as a strong predictor of poor health outcomes in elderly ${ }^{3-5}$ and adult patients with chronic disease. ${ }^{6-8}$

Both pharmacological and non-pharmacological interventions, such as psychotherapy or supervised exercise intervention, have been accepted as standard treatments of depression. However, concerns about drug side effects and 
dependency appear to make patients prefer psychological interventions. One study showed that around $70 \%$ of patients with depression were non-compliant with antidepressants because of concerns about their side effects. ${ }^{9}$ Even though non-pharmacological interventions were preferred over antidepressants, ${ }^{10}{ }^{11}$ only a very small percentage of patients referred for psychotherapy were able to enter and complete this treatment. ${ }^{12}$ This inconsistency between patient preference and low rates of initiation and adherence to treatment could be a consequence of barriers to obtaining treatment. Non-pharmacological interventions are usually clinic-based or hospital-based and require visits on a weekly or monthly basis. In one study, $70 \%$ of patients reported structural barriers preventing them from attending psychotherapy sessions regularly and cited time constraints, transportation problems and cost as being significant obstacles. ${ }^{13}$

Among older patients, whose prevalence rate of depression is very high, these problems were aggravated by concurrent medical illness, social isolation, functional impairment or being home-bound. ${ }^{14-20}$ Overcoming these barriers by providing interventions in patients' own homes may achieve better treatment adherence and thereby greater treatment success than clinic-based or hospital-based interventions.

Home-based non-pharmacological interventions, such as problem-solving therapy ${ }^{21-25}$ and home-based exercise intervention, ${ }^{26}{ }^{27}$ have developed over several years. Although some studies have suggested that these approaches can improve depressive symptoms and rates of remission when compared with standard usual care, ${ }^{26} 2829$ other studies have reported conflicting results. ${ }^{27} 3031$ In recent years new home-based interventions have been introduced to treat depression, such as spirituality teaching programme ${ }^{32}$ and combined homebased psychotherapy with exercise intervention, but their efficacy is still controversial. Therefore, our systematic review and network meta-analysis were undertaken with the aim of reviewing all available home-based non-pharmacological interventions, pooling the effect sizes of each intervention on symptom improvement and indirectly comparing treatment efficacy between the different interventions. The results of this review should be useful for identifying the most beneficial home-based non-pharmacological interventions and for informing clinical guidelines for treating depression.

\section{METHODS}

\section{Search strategy}

Relevant studies were identified using Medline, Scopus and Cumulative Index to Nursing and Allied Health Literature (CINAHL) databases searched from inceptions to 7 August 2016. Reference lists of included studies were also explored. Search terms and search strategies for each database are presented in the online supplementary appendix 1.

\section{Selection of studies}

Initially studies were selected from titles and abstracts by two independent reviewers (KS and TA). Full articles were retrieved to aid decision making if decision could not be made based on titles and abstracts. Disagreement between the two reviewers was resolved by discussion. Percentage agreement between the two reviewers was estimated using kappa statistics.

\section{Inclusion criteria}

Randomised controlled trials published in English were eligible for the review if they met all of the following criteria:

1. Study participants were adults aged more than 18 years with a diagnosis of any degree of depressive disorder using the criteria of the Diagnostic and Statistical Manual of Mental Disorders 4th Edition (DSM-IV) or any diagnostic tool used for diagnosis or screening for depression. Participants who were children, adolescents or postpartum women were excluded.

2. Interventions of interest were non-pharmacological and provided in the patient's home, such as cognitive behavioural therapy (CBT), problemsolving therapy, family therapy or home-based exercise intervention.

3. Treatment comparison was the care of depression in outpatient clinics or hospital settings.

4. Outcomes were measured by the level or severity of depressive symptoms or the incidence of disease remission at the end of the intervention.

5. Studies provided sufficient data for analysis, such as number of participants (n), mean depression score, $\mathrm{SD}$ for each intervention group and the number of patients per intervention group with or without disease remission.

\section{Data extraction}

Two reviewers (KS and TA) independently used a standardised data record form to extract baseline characteristics of included studies and outcomes of interest. Disagreement between the two reviewers was resolved by discussion, and corresponding authors of the studies were contacted if information was incomplete.

\section{Interventions of interest}

Home-based non-pharmacological intervention was defined as any care or management of depression provided by healthcare professionals at a patient's place of residence. Home-based interventions had to have a clear and definite objective. Home visits that provided only health education, social or emotional support were, therefore, excluded from this study.

Web or internet-based interventions were excluded from this review due to their variability in terms of content, accessibility, usability, methods of delivery and supplementary tools. ${ }^{33}$ 


\section{Outcomes of interest}

The outcomes of interest were depressive symptom scores and disease remission rates at the end of treatment. Disease remission was defined according to the criteria of the original article. Included studies used several tools for measuring the severity of depressive symptoms, namely the Hamilton Depression Rating Scale (HAM-D), ${ }^{21} 22303435$ Patient Health Questionnaire-9 (PHQ-9), ${ }^{24} 36$ Geriatric Depression Scale (GDS), ${ }^{23} 2628$ Hopkins Symptom Checklist-20 (HSCL20), ${ }^{37} 38$ Montgomery-Asberg Depression Rating Scale (MADRS) ${ }^{18}{ }^{25}{ }^{27}$ Beck Depression Inventory-Fast Screen $(\text { BDI-FS })^{39}$ and Center of Epidemiologic Studies Depression Scale (CES-D). ${ }^{40}$ These tools have different score ranges (HAM-D $=0-53$, PHQ-9 $=0-27$, GDS $=0-15$, HSCL$20=0-4$, MADRS $=0-60$, BDI-FS $=0-21$ and CES-D $=0-60$ ), with higher scores in all tools representing increasing severity of depressive symptoms.

\section{Risk of bias assessment}

To assess the quality of included studies, a risk of bias assessment tool $^{41}$ was applied by two independent reviewers (KS and TA). Seven domains were evaluated as follows: (1) random sequence generation, (2) allocation concealment, (3) blinding of participants and personnel, (4) blinding of outcome assessors, (5) incomplete outcome data, (6) selective outcome reporting and (7) other sources of bias. The quality of the studies was classified as being at high, unclear or low risk of bias. We added the domain of power to determine the likelihood of any studies making a true difference to outcome, classifying any study as being at high risk of bias if it reported a power less than $80 \%$. Disagreement between the two reviewers was settled by discussion.

\section{Statistical analysis}

Because depression scores were measured differently among the studies, for direct comparison the standardised mean differences (SMD) of depressive scores between intervention and control groups were estimated for individual studies and then were pooled across studies. Heterogeneity between studies was estimated by $\mathrm{Q}$ test and $\mathrm{I}^{2}$ statistic. Heterogeneity between studies was considered if the $p$ value from $Q$ test was less than 0.10 or if $\mathrm{I}^{2}$ was equal to or greater than $25 \% .^{42}$ If heterogeneity was presented, the SMD was estimated by applying the random-effect model. Otherwise the fixed-effect model was applied.

For dichotomous outcomes, relative risks (RR) of disease remission were calculated for each study. The random-effect model was used for pooling RR if there was evidence of heterogeneity between studies. Otherwise the inverse variance method was used. Sources of heterogeneity were explored by considering possible factors one by one in a meta-regression model (eg, mean age, severity of depression at baseline and types of intervention delivery).
A network meta-analysis was applied to indirectly assess intervention effects for all home-based interventions, that is, home-based psychological intervention, home-based exercise intervention, combined home-based psychological intervention and exercise intervention, and usual care. This method allows us to perform indirect comparison using common comparator. For instance, some studies compared home-based psychological intervention with usual care, some others compared home-based exercise intervention with usual care, and none or few studies compared home-based psychological intervention with home-based exercise intervention. Using common comparator as usual care would allow to indirectly compare home-based psychological intervention with home-based exercise intervention. ${ }^{43}$ Treatment effects for each study were estimated using a two-stage network meta-analysis. First, summary data were expanded into individual patient data using the 'expand' command in Stata V.14 program. Binary regression was applied to estimate $\log (\mathrm{RR})$ and variance-covariance of each treatment using 'mvmeta' make command. A multivariate random-effect meta-analysis was used to calculate the pooled RRs and their 95\% CIs. Riley's method was used for considering subject-study correlation. Treatment ranking was made according to the linear predictor of each study. In addition, a consistency assumption (ie, discrepancy of intervention effects between direct and indirect meta-analyses) was assessed using the standardised normal test $(Z)$.

Publication bias was assessed using Egger's test and funnel plot. If the funnel plot showed asymmetry, a contour-enhanced funnel plot was performed to explore whether asymmetry was the result of heterogeneity between studies or arisen from publication bias. All analyses were performed using Stata V.14. A two-sided test with $p$ value less than 0.05 was considered statistically significant, except for the $Q$ test, in which a $p$ value less than 0.10 was applied.

\section{Patient involvement}

Patients were not involved in the design of the study, development of outcome measures or conduct of the study. We did not ask patients for advice on interpreting or writing up the results. There are no plans to disseminate the results of the research to study participants.

\section{RESULTS}

We identified 385, 534, 255 and 2 articles from Medline, Scopus, CINAHL databases and reference lists, respectively. After deleting duplications, the titles and abstracts of 768 studies were reviewed. Finally, 17 studies met our inclusion criteria and were eligible in the review (figure 1). Agreement of study selection between the two reviewers was high at $86.7 \%(\mathrm{kappa}=0.50)$. All but one ${ }^{18}$ of the included studies reported protocol approval by an ethics committee. 


\section{Study participants}

The baseline characteristics of included studies are presented in table 1 . The type and severity of depression of participants differed between studies. Four studies ${ }^{25} 283435$ included patients with major depressive disorder (MDD), four 22233840 included patients with minor depression and nine 182124262730363739 included patients with mixed severities of depression. Ten studies included elderly patients, ${ }^{18} 21-26283840$ while seven studies included adults with ages greater than 18years ${ }^{27} .3034-3739$ Ten studies included patients with depression with other comorbidities (ie, epilepsy, ${ }^{37}$ heart diseases, ${ }^{22} 3036$ disability ${ }^{18}$ and mild to moderate cognitive impairment ${ }^{25}$ ). Twelve studies $^{21} 23$ 24 26-28 34 35 38-40 included patients without comorbidity. Use of antidepressants at baseline varied widely between studies, ranging from $0 \%$ to $95 \%$.

\section{Home-based interventions}

The composition of home-based non-pharmacological interventions differed among the included studies but

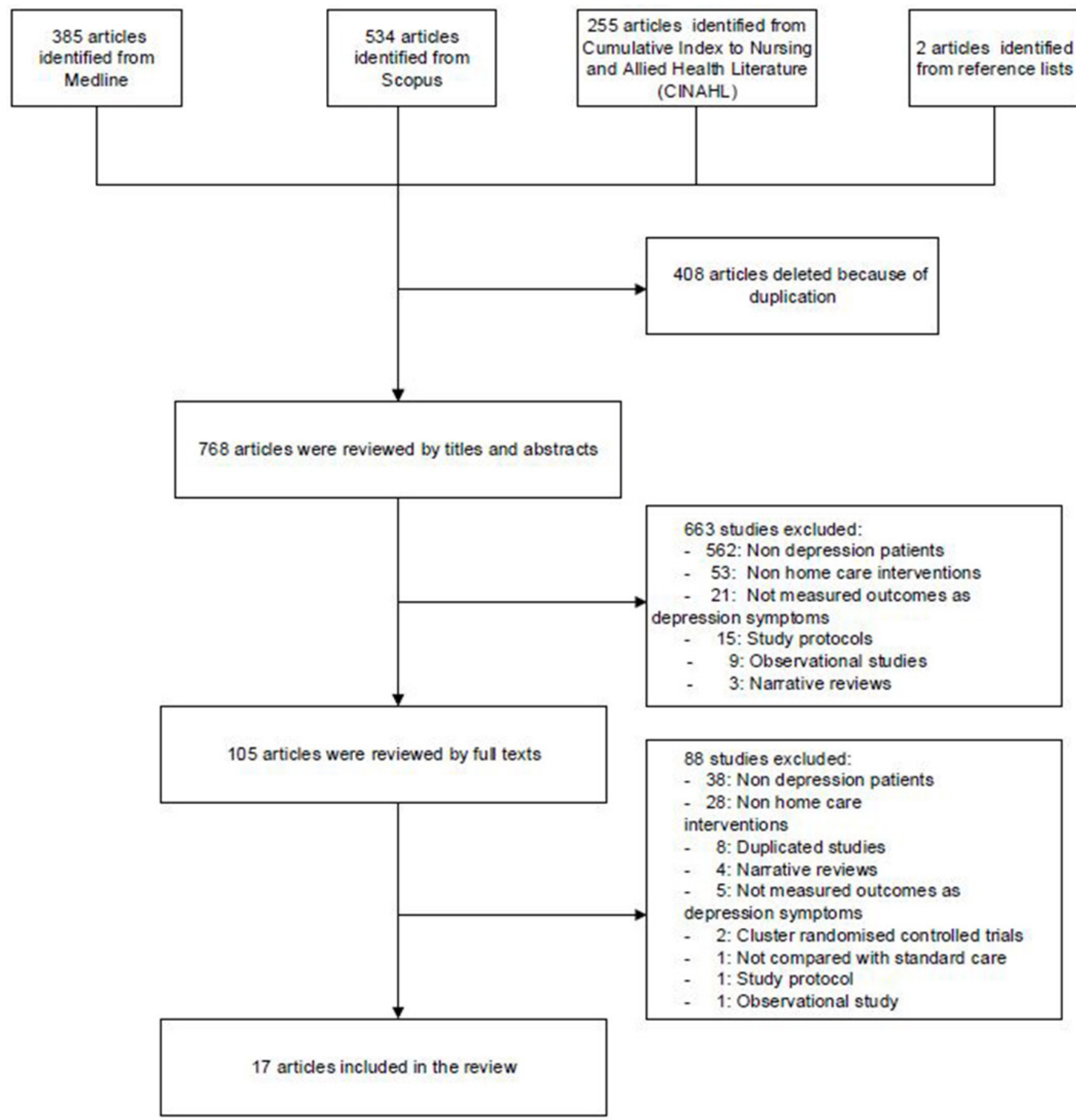

Figure 1 Flow chart of study selection. 


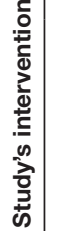

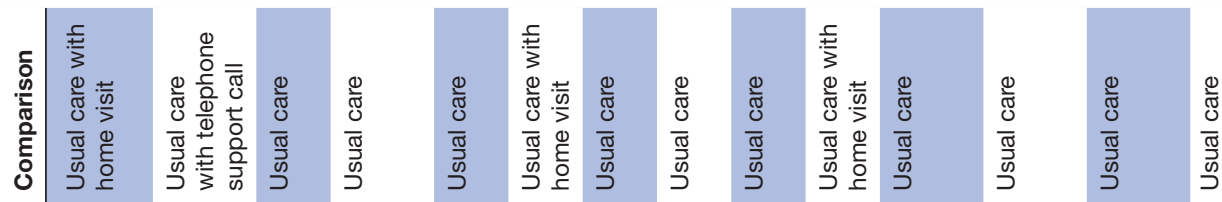

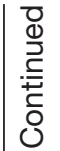

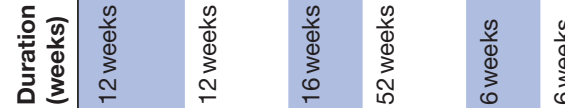

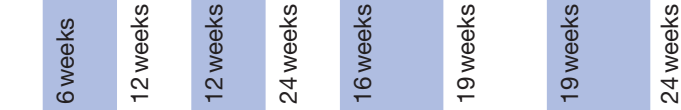

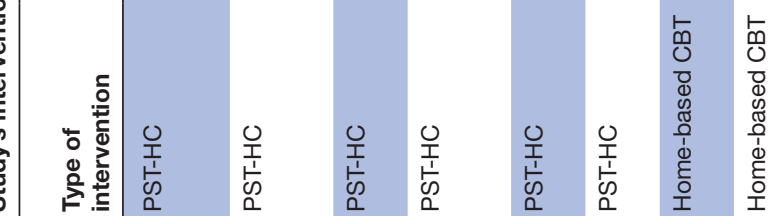

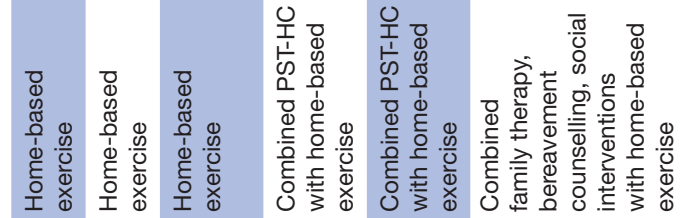

宽

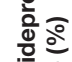

击离

竞

$\stackrel{0}{\stackrel{0}{2}}$

$\frac{0}{0}$

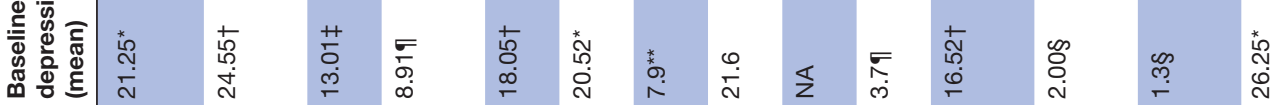

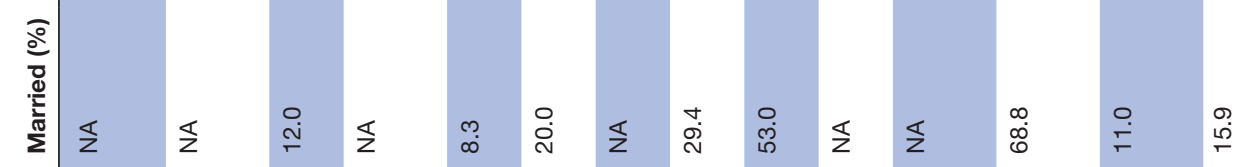

$\stackrel{9}{\frac{0}{\sigma}}$

:

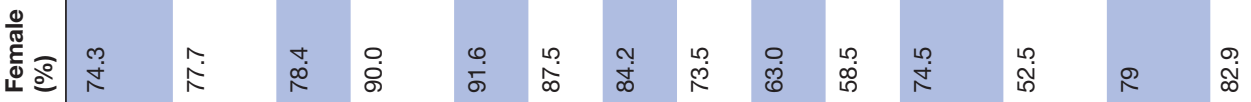

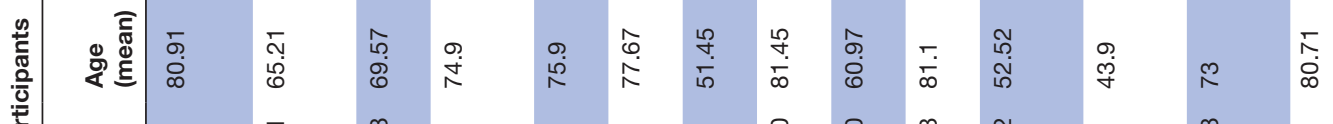

$\mathbf{z}$ ป

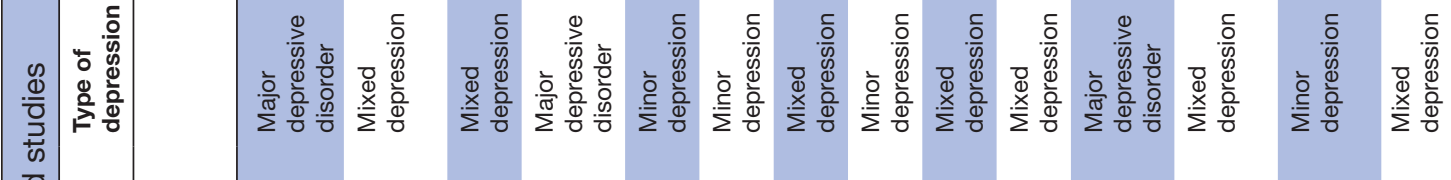
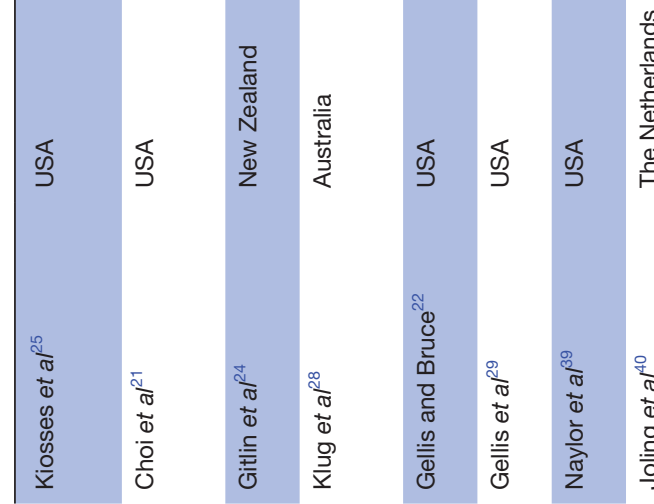

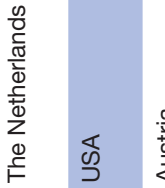
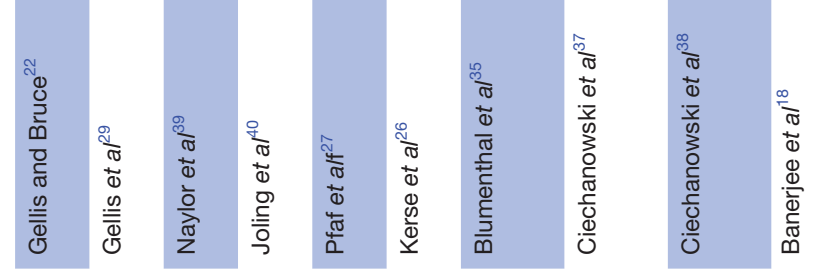


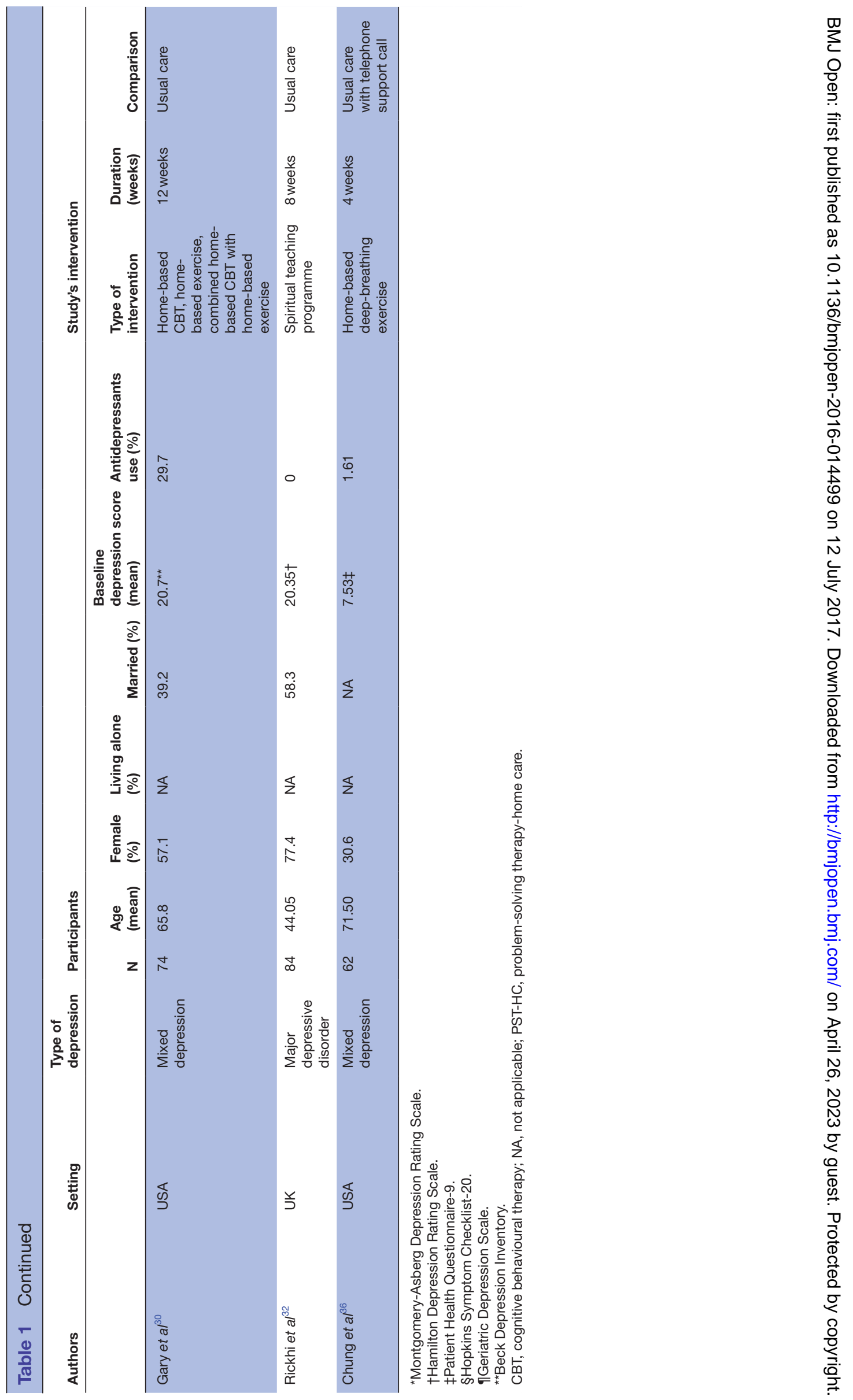


could be categorised into four groups: (1) home-based psychological intervention, (2) home-based exercise intervention, (3) combined home-based psychological intervention with home-based exercise intervention and (4) complementary medicine. Eight, three and three studies compared home-based psychological intervention with usual care, home-based exercise intervention with usual care and combined home-based psychological intervention with home-based exercise intervention with usual care, respectively. One study compared the efficacy between home-based psychological intervention, homebased exercise intervention, combined home-based psychological intervention with home-based exercise intervention and usual care. Since interventions in the category of complementary medicine were heterogeneous, they were not included in the meta-analysis but were subjected to qualitative analysis. Details of each home-based intervention are summarised below.

\section{Home-based psychological intervention}

Home-based psychological intervention was classified as home-based problem-solving treatment (PST) or homebased CBT.

\section{Home-based PST (six studies 212224252829 )}

Home-based PST is a skill-enhancing behavioural treatment of depression usually delivered by social workers and psychologists. This approach assumes that depressive symptoms are caused and maintained by problems of daily life and that these can be reduced by identifying and addressing them systematically. Each PST session comprises (1) defining and formulating the nature of the depressive problem, (2) generating a range of alternative solutions to the problem, (3) systematically evaluating the possible consequences of each solution then selecting the most appropriate one and (4) monitoring and evaluating the actual outcome. In addition, PST identifies patients' pleasurable activities and encourages them to participate in these activities.

\section{Home-based CBT (three studies ${ }^{303940}$ )}

The aim of CBT is to modify the dysfunctional emotions, behaviours and thoughts of patients with depression. This type of intervention was identified in three studies, of which two studies were CBT-based bibliotherapy. In this approach, participants received self-help books or leaflets that included instruction on cognitive behavioural self-help, mood management skills and tasks to practise. During the intervention period, participants were visited by home care nurses or contacted by telephone by study investigators to assess their symptoms and encourage them to follow the course at their own place.

\section{Home-based exercise intervention}

Home-based exercise intervention was employed in four studies. Exercise included both progressive resistance training and aerobic exercise $\mathrm{e}^{2627}$ and aerobic exercise only. ${ }^{30}$ Participants were required to perform exercises of moderate intensity for at least $30 \mathrm{~min}$ three times a week.

Combined home-based psychological intervention with exercise intervention

Home-based psychological intervention combined with exercise intervention was the intervention of interest in four studies. Each of these applied aerobic exercise as a home-based exercise intervention but psychological therapies differed among them (PST in two studies, ${ }^{37} 38$ CBT in one study ${ }^{30}$ and family therapy with bereavement counselling and social interventions in one study ${ }^{18}$ ).

\section{Complementary medicine}

This intervention refers to a broad set of healthcare practices or activities that are not integrated into the dominant healthcare system. This type of intervention was used in two studies. One involved home-based deepbreathing exercise ${ }^{36}$ aimed at stimulating a relaxation response (ie, to decrease arousal, heart rate and blood pressure, and to reduce responsiveness of the sympathetic nervous system). Deep-breathing exercises can also help patients to disregard negative and distracting thoughts. Patients were trained by experienced nurses to breathe at a rate of six breaths per minute. During the treatment period, this was undertaken in a quiet environment for a period of 10 min three times each day.

The other intervention employed a spirituality teaching programme ${ }^{32}$ aiming to promote contemplation of the interrelation between meaning and purpose, connectedness with others, nature or the divine, and values such as compassion, love, justice and forgiveness. The programme comprised eight sessions involving explanation of the divine aspect of the self, teaching breathing and visualisation practice, helping patients to connect with the divine through prayer or meditation, letting go of regret and fostering gratitude, practising self-awareness relating to the five senses and building upon connectedness with others. Participants were also advised to avoid forming expectations and to refrain from judging outcomes. Initially patients participated in a workshop run by psychiatrists and were then required to practise by themselves with the help of audio CDs.

\section{Treatment comparison}

Usual care was defined as the care of depression managed by healthcare providers (ie, general practitioners, psychiatrists, nurses or social workers) and could include onward referral to appropriate treatment services if specified in the study protocol. In addition to usual care, three studies $^{252629}$ included home visits and two ${ }^{21} 36$ included telephone support. However, these interventions provided education about depression together with general social or emotional support but did not offer specific psychological help or exercise intervention. With the exception of one study, ${ }^{32}$ almost all used antidepressant medication as a cointervention, but the decision to initiate or maintain 
this was dependent on the judgements of patients and their physicians.

\section{Risk of bias assessment}

Results of risk of bias assessment are presented in online supplementary table 1 . Most of the studies (14 studies) reported low risk of bias in the domain of random sequence generation, while three studies ${ }^{21} 2230$ reported unclear risk. For allocation concealment, 11 studies $^{18} 212225-303940$ had unclear risk of bias, while 6 studies $^{24} 32$ 35-38 had low risk of bias. All studies reported high risk of bias in the domain of blinding of participants and personnel and low risk of bias in other domains. Ten studies ${ }^{182225-272930323537}$ had low risk of bias for blinding of outcome assessors, whereas seven studies $2124283638-40$ had unclear risk. For the domain of incomplete outcome data, 13 studies ${ }^{182224-2729303235373840}$ reported low risk of bias and 4 studies 21283639 reported high risk. Almost all studies (16 studies) had low risk of bias for selective outcome reporting, while only one study ${ }^{37}$ had high risk of bias. In the domain of power, 10 studies had unclear risk of bias, 6 reported low risk and 1 had high risk of bias.

\section{Pooled mean difference of depression score}

Home-based psychological intervention versus usual care

Nine studies were analysed ${ }^{21} 22242528-303940$ comparing home-based psychological intervention with usual care $(n=739)$. Depression scores were assessed at the end of treatment, which ranged from 6 to 48 weeks. The mean differences and 95\% CIs of depression scores for each study are presented in table 2. The SMD of home-based psychological intervention versus usual care was -0.57 (95\% CI -0.84 to -0.31 ), suggesting that home-based psychological intervention can significantly decrease depression scores when compared with usual care.

Moderate heterogeneity was found between studies, with $\mathrm{I}^{2}$ of $63.5 \%$. Sources of heterogeneity were explored but none of the factors decreased $\mathrm{I}^{2}$. Subgroup analyses were performed according to the severity of depression (ie, MDD, minor depression and mixed severity of depression). These showed that SMDs in patients with MDD, minor depression and mixed severity of depression were -0.95 (95\% CI -1.35 to $\left.-0.55 ; \mathrm{I}^{2}=16.5 \%\right),-0.61 \quad(95 \%$ CI -1.28 to $\left.0.06 ; \mathrm{I}^{2}=82.1 \%\right)$ and $-0.41,(95 \%$ CI -0.62 to $-0.19 ; \mathrm{I}^{2}=0 \%$ ), respectively (see online supplementary figure 1). These suggest that home-based psychological intervention reduces depression scores significantly in the groups of MDD and mixed severity of depression. In addition, the efficacy of this intervention in patients with MDD was better than the efficacy in patients with minor depression.

Home-based exercise intervention versus usual care

Three studies ${ }^{26} 3035$ ( $\left.n=321\right)$ were pooled to compare the effect of home-based exercise intervention with usual care. The SMD was -1.03 (95\% CI -2.89 to 0.82 ; $\mathrm{I}^{2}=97.9 \%$ ) (table 2 and online supplementary figure $2 \mathrm{~A}$ ). The mean depression score in the home-based exercise intervention group was therefore 1.03 units lower than the mean depression score in the usual care group. However, this effect did not reach statistical significance.

Combined home-based psychological intervention with exercise intervention versus usual care

Only two studies ${ }^{38}(\mathrm{n}=169)$ had sufficient data for pooling the effect on the depression score of combined home-based psychological intervention with exercise intervention. Mean depression scores were measured at the ends of the treatments, namely at 12 weeks $^{30}$ and 24 weeks. ${ }^{38}$ Table 2 shows the mean depression scores and $95 \%$ CIs for each study. The SMD was $-0.78(95 \%$ CI -1.09 to $-0.47 ; \mathrm{I}^{2}=0.0 \%$ ) (online supplementary figure 2B). This indicates that patients receiving combined home-based psychological intervention and exercise intervention had significantly lower mean depression scores (by 0.78 units) than patients receiving usual care.

\section{Pooled risk ratio of disease remission}

Home-based psychological intervention versus usual care

Four studies $(n=459)$ comparing the effectiveness of home-based psychological intervention with usual care had remission rates as their outcome of interest. Remission from depression was defined as HAMD score $\leq 7$ in one study, ${ }^{25}$ PHQ- $9 \leq 4$ in one study, ${ }^{24} \mathrm{BDI}<4$ in one study ${ }^{39}$ and CES-D $<16$ in one study ${ }^{40}$ (table 3 ). The pooled RR was 1.53 (95\% CI 1.19 to 1.98) (online supplementary figure 3), suggesting that patients receiving home-based psychological intervention were approximately 1.7 times more likely to have remission from depression than patients receiving usual care. The results among studies were homogeneous with an $\mathrm{I}^{2}$ of $0 \%$.

\section{Home-based exercise intervention versus usual care}

Two studies $(\mathrm{n}=248)$ reported their outcome as disease remission and had sufficient data for pooling. Disease remission was defined as HAMD score $\leq 7^{35}$ or symptoms no longer meeting the criteria for major and minor depression according to the DSM-IV criteria ${ }^{27}$ (table 3 ). The pooled RR was 0.99 (95\% CI 0.79 to $\left.1.24 ; \mathrm{I}^{2}=0.0 \%\right)$ (online supplementary figure $4 \mathrm{~A}$ ), indicating that there was no significant difference between home-based exercise intervention and usual care in the likelihood of having remission from depression.

\section{Combined home-based psychological intervention and exercise intervention versus usual care}

Three studies $(\mathrm{n}=279)$ were pooled to compare remission rates between combined psychological intervention and usual care. Disease remission was defined as HSCL-20 score $<0.5$ for two studies, ${ }^{3738}$ but in Banerjee $e t a l$ s study ${ }^{18}$ the outcome was not clearly defined (table 3 ). Pooled RR was 3.47 (95\% CI 2.11 to $5.70 ; \mathrm{I}^{2}=19.7 \%$ ), suggesting that the combination of home-based psychological intervention and exercise intervention significantly increased the likelihood of remission from depression when compared with usual care (online supplementary figure 4B). 


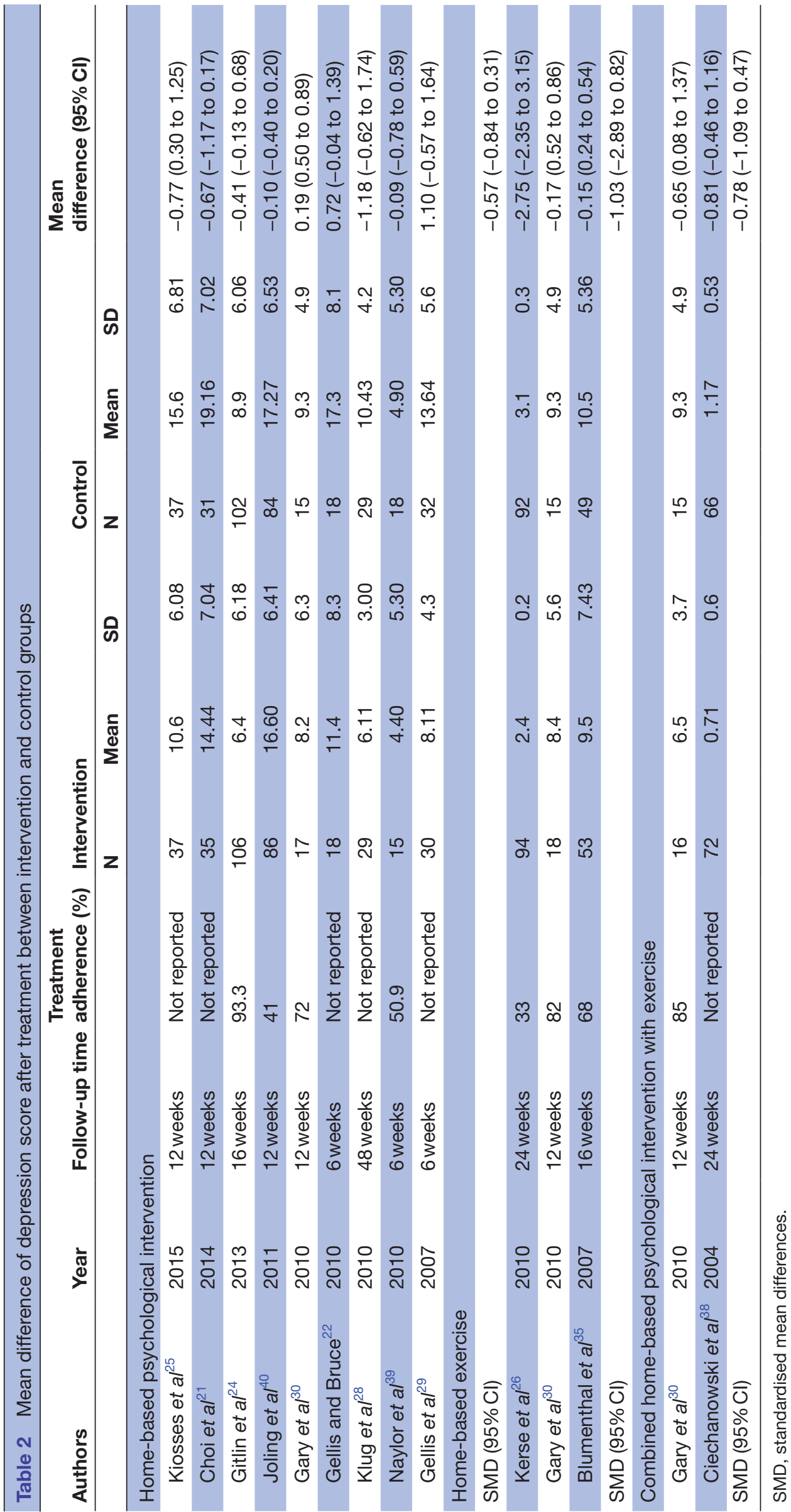




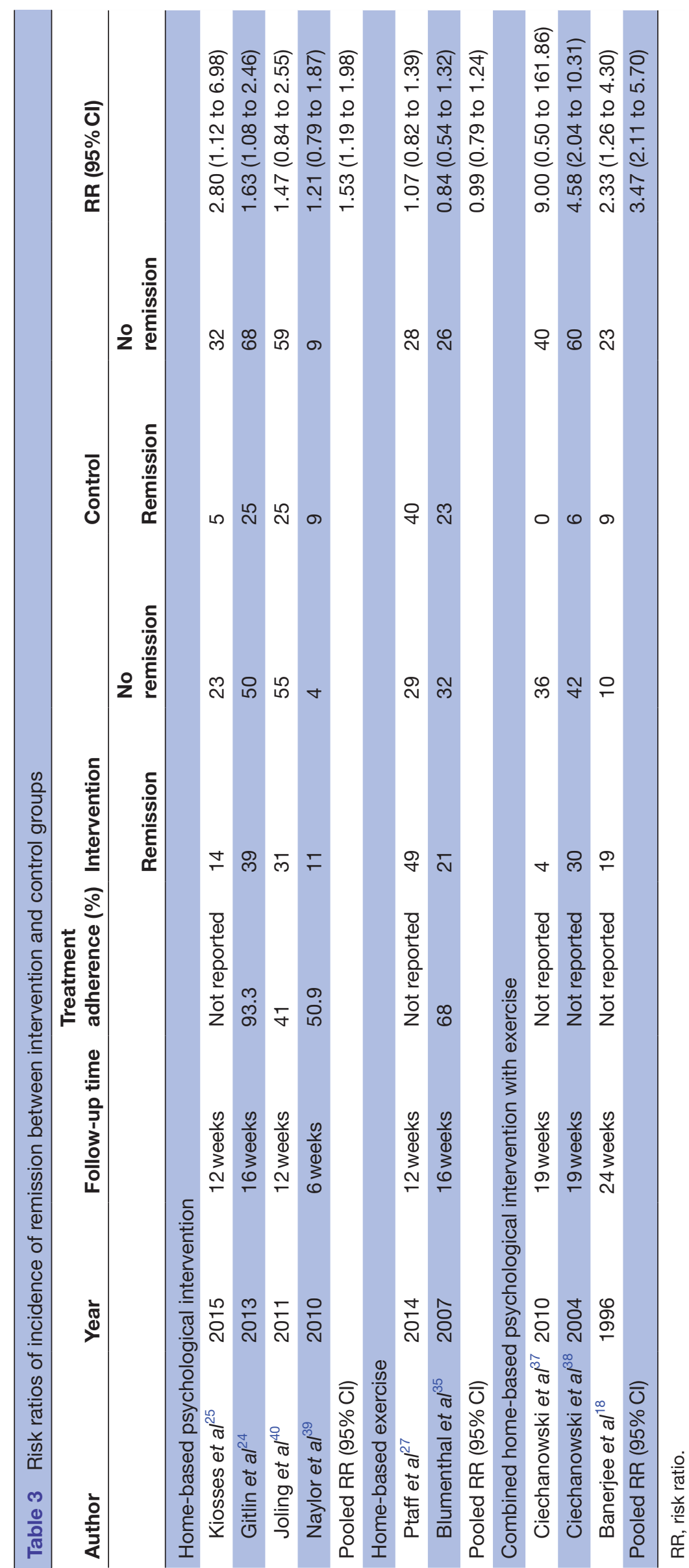

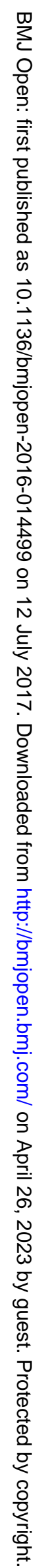




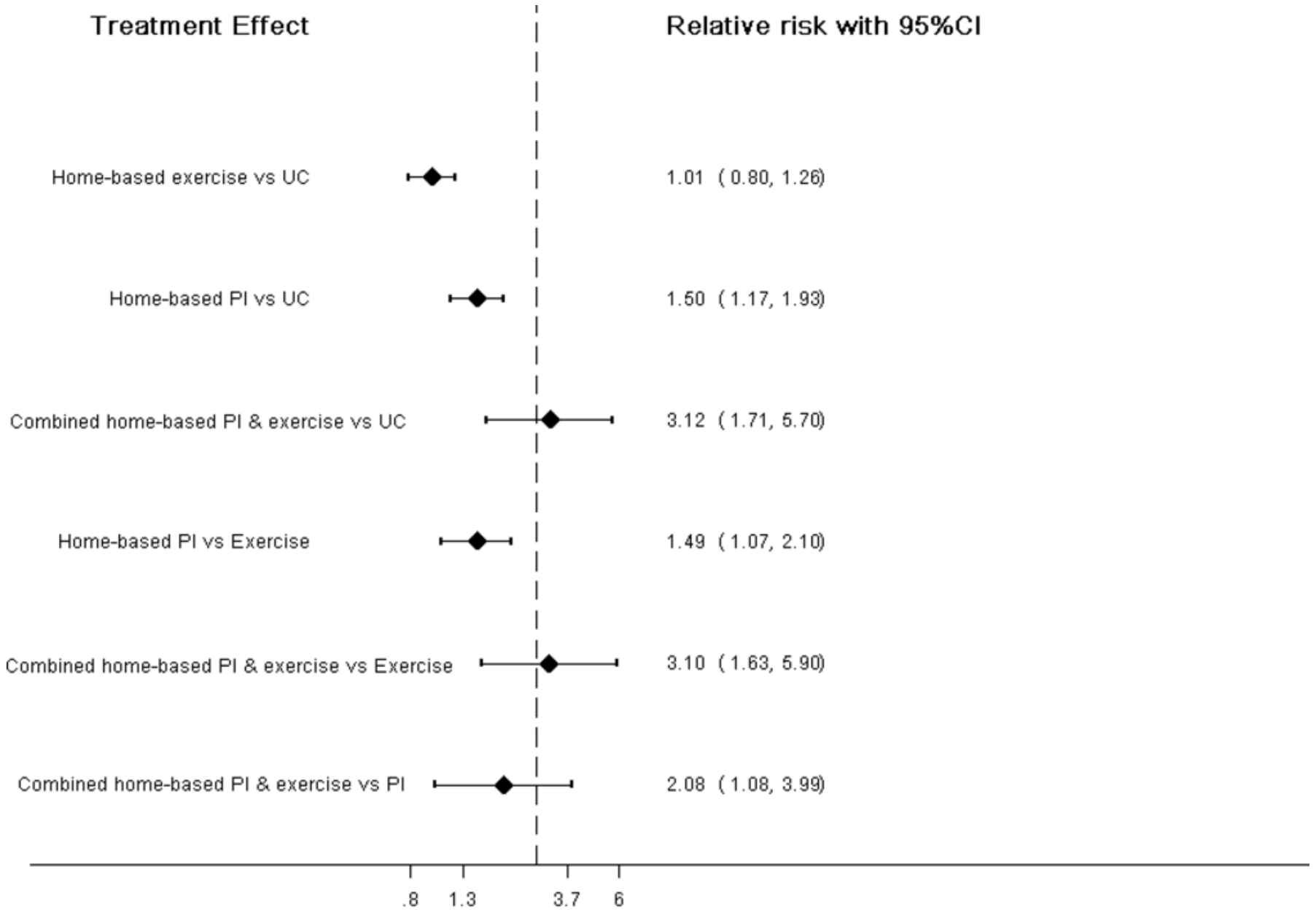

$\mathrm{PI}$, psychological intervention; UC, usual care

Figure 2 Network meta-analysis of disease remission among home-based interventions.

\section{Network meta-analysis}

Disease remission rate

Nine studies $(\mathrm{n}=987)$ were included in the network meta-analysis. Online supplementary figure 5 shows the network plot of home-based psychological intervention, home-based exercise intervention, combined home-based psychological intervention with exercise intervention and usual care. Size of node and edge reflect the number of studies and patients, respectively. From the plot, usual care was the common comparator and had the largest sample size of the four treatment regimens. Home-based psychological intervention versus usual care had the largest number of studies.

Pooled RRs for each treatment comparison are presented in figure 2. Compared with usual care, only combined home-based psychological intervention with exercise intervention and home-based psychological intervention alone significantly increased the likelihood of disease remission, with pooled RRs of 3.12 (95\% CI 1.71 to 5.70 ) and 1.50 (95\% CI 1.17 to 1.93 ), respectively. In addition, the incidence of disease remission in home-based psychological intervention and combined home-based psychological intervention with exercise intervention groups was significantly higher than in the home-based exercise intervention group, having pooled RRs of 1.49 (95\% CI 1.07 to 2.10) and 3.10 (95\% CI 1.63 to 5.90), respectively. When compared with home-based psychological intervention alone, combined home-based psychological intervention with exercise intervention also significantly improved the rate of disease remission (pooled RR=2.08; 95\% CI 1.08 to 3.99).

Treatment ranking was assessed by estimating the probability of each treatment being the best. This yielded probabilities of $99.5 \%, 0.5 \%$ and $0 \%$ for combined home-based psychological intervention with exercise intervention, home-based psychological intervention alone and home-based exercise intervention, respectively. Combined home-based psychological intervention with exercise intervention therefore emerged as the best intervention for achieving remission from depression.

Applying inconsistency assumptions to the treatments, inconsistency factors were calculated as $0.020(\mathrm{Z}=0.183$, $\mathrm{p}=0.912)$ for home-based psychological intervention versus usual care, $-0.018(\mathrm{Z}=0.110, \mathrm{p}=0.913)$ for homebased exercise intervention versus usual care, and 0.106 $(\mathrm{Z}=0.107, \mathrm{p}=0.915)$ for combined home-based psychological intervention with exercise intervention versus usual care. These figures find no significant difference between 
the direct and indirect comparisons of estimated treatment effects.

\section{Efficacy of complementary medicine}

Results from a study comparing the efficacy of homebased deep-breathing exercises with usual care showed that patients receiving this intervention had significantly lower depression scores than patients receiving usual care, with a mean difference of -1.34 (95\% CI -1.17 to -0.17). Another study compared the efficacy of homebased spiritual therapy with usual care. Findings from this study suggested that home-based spiritual therapy could significantly decrease depression scores when compared with usual care, with a mean difference of -1.11 (95\% CI -1.57 to -0.65$)$. In addition, this study found that patients receiving home-based spiritual therapy were more likely to have disease remission than patients receiving usual care. The risk ratio of disease remission (defined as HAMD score $\leq 7)$ from this study was 13.85 (95\% CI 1.88 to 101.74 )

\section{Publication bias}

Egger's tests and funnel plots did not suggest any publication bias for pooling the effect of home-based psychological intervention (coefficient $=-0.05, \mathrm{p}=0.882$ ) and home-based exercise intervention (coefficient $=6.94$, $\mathrm{p}=0.818$ ) (see online supplementary figure $6 \mathrm{~A}, \mathrm{~B}$ ). For combined home-based psychological intervention and exercise intervention, Egger's test did not suggest publication bias, but a funnel plot showed asymmetry (online supplementary figure $7 \mathrm{~A}$ ). This inconsistency might be due to Egger's test having insufficient power to detect a difference when only three studies were considered in the analysis.

The cause of this asymmetry was further explored by performing a contour-enhanced funnel plot, which showed that most of the studies fell inside the significant area. The asymmetrical plot may, therefore, result from a small study effect rather than heterogeneity between studies (see online supplementary figure $7 \mathrm{~B}$ ).

\section{DISCUSSION}

Our study found that a combination of home-based psychological intervention with exercise intervention and home-based psychological intervention alone both significantly decreased depressive symptoms and increased the likelihood of disease remission when compared with usual care. The SMD of home-based psychological intervention versus usual care was -0.57 , which reflects the moderate magnitude of treatment effect, while the SMD of combined home-based psychological intervention with exercise intervention versus usual care was -0.78 , revealing the high magnitude. ${ }^{44}$ Treatment effect of these two interventions was comparable with selective serotonin reuptake inhibitor, which had an SMD of 0.05 , for treatment of depression. ${ }^{45}$ However, we could not demonstrate any benefit of home-based exercise intervention alone when compared with usual care. In addition, combined home-based psychological intervention with exercise intervention had the highest probability of remission from depression compared with both home-based psychological intervention and home-based exercise intervention.

The effectiveness of clinic-based psychological intervention for treating depression has been confirmed by previous studies. ${ }^{406}$ The results of our study also support the efficacy of this intervention when performed in the patient's home. In our review, the majority of participants in the included studies were patients with depression with comorbidities such as epilepsy ${ }^{37}$ or heart disease, ${ }^{22} 3036$ or were elderly patients with disabilities ${ }^{18}$ or cognitive impairment. ${ }^{25}$ These groups have a high prevalence of depression and should therefore be expected to receive a significant share of mental health provision. However, their ability to access conventional clinic-based mental health services is restricted by mobility problems and low motivation to seek help. With its ability to overcome these barriers, home-based psychological intervention is particularly appropriate in these situations.

In addition to demonstrating the efficacy of homebased psychological intervention overall, subgroup analysis within our study raises an interesting point. Home-based psychological intervention decreased symptoms of depression for all types of depression, but the effect was statistically significant only in patients with MDD and mixed severity depression, not for those with minor depression. This inconsistent finding may result from the so-called 'ceiling' effect. The level of depressive symptoms in minor depression is relatively low at baseline when compared with major depression, which could limit the potential for symptom improvement. ${ }^{47}$ This ceiling effect was also found in the studies reviewing the use of antidepressant medication for minor depressive symptoms.

The rationale for home-based exercise intervention rests on the theory that increasing physical activity can improve depressive symptoms through psychological and physiological routes. Exercise serves as a distraction from worries and depressive thoughts, increases self-efficacy and gives a sense of mastery. Possible physiological mechanisms include an increase in body temperature leading to a feeling of relaxation and reduced muscle tension, an increase in levels of endorphins related to positive mood and a sense of well-being, increases in the availability of the central neurotransmitters dopamine, norepinephrine and serotonin, and changes in neurobiological response. ${ }^{48-50}$ Although previous literature has demonstrated the benefit of supervised exercise in treating depression, ${ }^{51}{ }^{52}$ our study found no benefit from home-based exercise intervention in ameliorating depressive symptoms. The differences in these findings may be explained by poorer treatment compliance in the home-based exercise intervention group, as to achieve significant benefit patients have to practise the exercise programme at a prescribed intensity and frequency. Lack of motivation and inattention are common symptoms in depression and may account for unsupervised patients 
failing to achieve the prescribed levels of activity when compared with those given encouragement through supervision. This assumption corresponds with findings from the home-based exercise studies ${ }^{26} 27$ that the physical health of patients in this group (ie, cardiorespiratory capacity, body mass index and lower limb strength), the surrogate endpoints of exercise intervention, did not change significantly from baseline.

Although our study did not find any advantages of home-based exercise intervention over usual care, when combined with psychological intervention the combined approach had a significantly greater benefit than either of these interventions alone. The combination may have a synergistic effect, with psychological intervention improving motivation to initiate and maintain an exercise programme, while the latter in turn enhancing the benefits of psychological intervention. ${ }^{53}$

Reviewing complementary medicine approaches, results from Chung et $a l^{36}$ and Rickhi et $a l^{22}$ showed that home-based deep-breathing training and spiritual teaching programmes could significantly reduce depressive symptoms when compared with usual care. However, these two studies had small sample sizes and included specific populations, namely patients with coronary heart disease for Chung et al's study and middle-aged women for Rickhi $e t a l$ s study. Their findings may not, therefore, be generalisable to other populations.

\section{Strengths and limitations}

Our study has several strengths. Given the potential of home-based interventions to treat depression and the increasing use of these interventions, our analysis provides the first comprehensive review of the efficacy of home-based interventions in treating depression. A comprehensive search was undertaken to identify as many relevant studies as possible, and two reviewers selected the studies independently with a high level of agreement. Selection bias was, therefore less likely. In addition, we performed a network meta-analysis to compare the efficacy of all available home-based interventions in order to identify the best treatment regimen.

We are aware that our study may have some limitations. First, the quality of included studies in the area of allocation concealment was not optimal. The results from our study might, therefore, be affected by selection bias and should be interpreted with caution. Second, participants in our included studies were aware both of their own interventions and their subjective outcomes, including the self-reported depression scores. However, in most of the included studies, this bias from non-blinded intervention was minimised by blinding the outcome assessors. Third, although all studies used usual care as a treatment comparator, descriptions of usual care differed among the included studies (see online supplementary table 2). For instance, usual care in Blumenthal et als study consisted of each participant in the control group being prescribed antidepressant medication, while home-based exercise participants received none. This may be responsible for a high remission rate in the control group, resulting in an underestimated treatment effect for home-based exercise.

Network meta-analysis allows indirect comparison of the efficacy of all possible interventions, given the presence of some common comparators. However, this technique requires two important assumptions, namely transitivity and consistency. ${ }^{54}$ Transitivity requires that the characteristics of patients and studies subjected to direct and indirect comparisons should be similar. For instance, the characteristics of patients in a study comparing homebased psychological intervention with usual care and of patients in a study of home-based exercise versus usual care should be similar in order to perform an indirect comparison of home-based psychological intervention versus home-based exercise. This assumption cannot be directly assessed or tested. However, consistency testing is a manifestation of transitivity. We therefore made a consistency assumption by comparing intervention effects between direct and indirect estimates. The number of included studies may play a role in this assessment, that is, a false-negative result might be present when direct and indirect estimates are not statistically different. For our study, inconsistency factors ranged from -0.018 to 0.106 , at which levels a false-negative result is unlikely.

We were unable to check consistency assumption in three of the indirect comparisons due to a lack of direct comparisons. For each of these studies, we therefore explored patient characteristics (mean age and types of depression), the duration of the intervention, the percentage of antidepressant use in the intervention and control groups, and the description of usual care (see online supplementary table 2), and found variations between studies and comparisons. For example, the definitions of usual care in the studies of combined home-based psychological intervention with exercise were not clearly defined, while the common definition of usual care in the studies into home-based psychological intervention was the standard treatment of depression by psychiatrists. This heterogeneity within and between interventions across included studies may impact both on the results and on the transitivity and consistency assumptions of our network met-analysis. Results from indirect comparison, for example, the efficacy of home-based psychological intervention and combined home-based psychological intervention with exercise versus homebased exercise alone, should therefore be interpreted with caution. Ideally, confirmation should be sought by undertaking further randomised controlled trials directly comparing these two interventions with home-based exercise.

\section{Clinical implication and further study}

Depression is a common disorder, particularly among the elderly and in those with a chronic disease. Many of these patients have difficulty accessing mental health services due to physical disabilities and transportation problems. Home-based interventions to treat depression 
have the potential to overcome these barriers and have been shown by our study to be effective. This information should prove helpful when designing clinical guidelines. However, there are obvious logistical differences between home-based treatments for depression and conventional clinic-based care. Our study has not investigated these aspects of treatment design and, in particular, has not considered manpower implications or transport costs. An economic evaluation study is needed before general implementation of a home-based care model can be recommended unequivocally.

Although a randomised controlled trial is considered to be the gold standard for therapeutic research, in a real-world setting this design may not be ideal for examining patient preferences and adherence to treatment. In this review, most of the studies $(n=10)$ did not report the degree of adherence with the prescribed intervention. Seven studies demonstrated the effect of adherence on outcomes but used different definitions to assess adherence. To maximise treatment efficacy, further studies should determine the effects of patient preferences and adherence to treatment for different approaches and modes of delivery, as well as examine the factors that influence preferences and adherence.

\section{CONCLUSION}

Our study has confirmed the efficacy both of home-based psychological intervention and combined home-based psychological intervention with exercise intervention in decreasing symptoms of depression and improving rates of remission. In addition, the combination of home-based psychological intervention and exercise intervention has the highest probability of being the best treatment out of all available home-based interventions. This approach should, therefore, be considered when formulating clinical guidelines for treating depression.

Contributors $\mathrm{KS}, \mathrm{Pl}$ and TA were involved in the conception and design of the review. KS and TA developed the search strategy, performed study selection, extracted data from included studies and analysed the data. KS, ML, PI, AD, AT and TA were involved in the interpretation and discussion of results. KS and TA drafted the manuscript. PI, AD, AT and TA revised it critically for important intellectual content. All authors approved the final version of the article. All authors had access to all of the data in the study and can take responsibility for the integrity of the data and the accuracy of the data analysis.

Funding This research did not receive a specific grant from any funding agency in the public, commercial or not-for-profit sectors.

Competing interests All authors have completed the ICMJE uniform disclosure form at www.icmje.org/coi_disclosure.pdf and declare no support from any organisation for the submitted work, no financial relationships with any organisations that might have an interest in the submitted work in the previous three years and no other relationships or activities that could appear to have influenced the submitted work.

Patient consent This study did not involve human subjects.

Provenance and peer review Not commissioned; externally peer reviewed.

Data sharing statement No additional data available.

Open Access This is an Open Access article distributed in accordance with the Creative Commons Attribution Non Commercial (CC BY-NC 4.0) license, which permits others to distribute, remix, adapt, build upon this work non-commercially, and license their derivative works on different terms, provided the original work is properly cited and the use is non-commercial. See: http://creativecommons.org/ licenses/by-nc/4.0/

(c) Article author(s) (or their employer(s) unless otherwise stated in the text of the article) 2017. All rights reserved. No commercial use is permitted unless otherwise expressly granted.

\section{REFERENCES}

1. Ferrari AJ, Charlson FJ, Norman RE, et al. Burden of depressive disorders by country, sex, age, and year: findings from the global burden of disease study 2010. PLoS Med 2013;10:e1001547.

2. Reddy MS. Depression: the disorder and the burden. Indian $J$ Psychol Med 2010;32:1-2.

3. Penninx BW, Guralnik JM, Ferrucci L, et al. Depressive symptoms and physical decline in community-dwelling older persons. JAMA 1998;279:1720-6.

4. Rovner BW, German PS, Brant LJ, et al. Depression and mortality in nursing homes. JAMA 1991;265:993-6.

5. Unützer J, Katon W, Callahan CM, et al. Collaborative care management of late-life depression in the primary care setting: a randomized controlled trial. JAMA 2002;288:2836-45.

6. Ciechanowski PS, Katon WJ, Russo JE. Depression and diabetes: impact of depressive symptoms on adherence, function, and costs. Arch Intern Med 2000;160:3278-85.

7. Frasure-Smith N, Lespérance F, Talajic M. Depression following myocardial infarction. impact on 6-month survival. JAMA 1993;270:1819-25.

8. Katon W, Ciechanowski P. Impact of major depression on chronic medical illness. J Psychosom Res 2002;53:859-63.

9. Hunot VM, Horne R, Leese MN, et al. A cohort study of adherence to antidepressants in primary care: the influence of antidepressant concerns and treatment preferences. Prim Care Companion J Clin Psychiatry 2007;9:91-9.

10. Churchill R, Khaira M, Gretton V, et al. Treating depression in general practice: factors affecting patients' treatment preferences. $\mathrm{Br} J \mathrm{Gen}$ Pract 2000;50:905-6.

11. Riedel-Heller SG, Matschinger H, Angermeyer MC. Mental disorders--who and what might help? Help-seeking and treatment preferences of the lay public. Soc Psychiatry Psychiatr Epidemiol 2005;40:167-74.

12. Jaycox LH, Miranda J, Meredith LS, et al. Impact of a primary care quality improvement intervention on use of psychotherapy for depression. Ment Health Serv Res 2003;5:109-20.

13. Mohr DC, Hart SL, Howard I, et al. Barriers to psychotherapy among depressed and nondepressed primary care patients. Ann Behav Med 2006;32:254-8.

14. Geerlings SW, Beekman AT, Deeg DJ, et al. Physical health and the onset and persistence of depression in older adults: an eight-wave prospective community-based study. Psychol Med 2000;30:369-80.

15. Schoevers RA, Beekman AT, Deeg DJ, et al. Risk factors for depression in later life; results of a prospective community based study (AMSTEL). J Affect Disord 2000;59:127-37.

16. Krishnan KR, George LK, Pieper CF, et al. Depression and social support in elderly patients with cardiac disease. Am Heart $J$ 1998;136:491-5.

17. Prince MJ, Harwood RH, Thomas A, et al. A prospective populationbased cohort study of the effects of disablement and social milieu on the onset and maintenance of late-life depression. the Gospel Oak Project VII. Psychol Med 1998;28:337-50.

18. Banerjee S, Shamash K, Macdonald AJ, et al. Randomised controlled trial of effect of intervention by psychogeriatric team on depression in frail elderly people at home. BMJ 1996;313:1058-61.

19. Bruce ML, Hoff RA. Social and physical health risk factors for first-onset Major depressive disorder in a community sample. Soc Psychiatry Psychiatr Epidemiol 1994;29:165-71.

20. Zozula R, Rosen RC, Jahn EG, et al. Recognition of sleep disorders in a community-based setting following an educational intervention. Sleep Med 2005;6:55-61.

21. Choi NG, Hegel MT, Marti N, et al. Telehealth problem-solving therapy for depressed low-income homebound older adults. Am J Geriatr Psychiatry 2014;22:263-71.

22. Gellis ZD, Bruce ML. Problem solving therapy for subthreshold depression in home healthcare patients with cardiovascular disease. Am J Geriatr Psychiatry 2010;18:464-74.

23. Gellis ZD, McGinty J, Tierney L, et al. Randomized controlled trial of problem-solving therapy for minor depression in home care. Res Soc Work Pract 2008;18:596-606.

24. Gitlin LN, Harris LF, McCoy MC, et al. A home-based intervention to reduce depressive symptoms and improve quality of life in 
older African Americans: A randomized trial. Ann Intern Med 2013:159:243-52.

25. Kiosses DN, Ravdin LD, Gross JJ, et al. Problem adaptation therapy for older adults with Major depression and cognitive impairment: A randomized clinical trial. JAMA Psychiatry 2015;72:22-30.

26. Kerse N, Hayman KJ, Moyes SA, et al. Home-based activity program for older people with depressive symptoms: dellite- A randomized controlled trial. Ann Fam Med 2010;8:214-23.

27. Pfaff JJ, Alfonso H, Newton RU, et al. ACTIVEDEP: A randomised, controlled trial of a home-based exercise intervention to alleviate depression in middle-aged and older adults. Br J Sports Med 2014;48:226-32.

28. Klug G, Hermann G, Fuchs-Nieder B, et al. Effectiveness of home treatment for elderly people with depression: randomised controlled trial. Br J Psychiatry 2010;197:463-7.

29. Gellis ZD, McGinty J, Horowitz A, et al. Problem-solving therapy for late-life depression in home care: A randomized field trial. Am J Geriatr Psychiatry 2007:15:968-78.

30. Gary RA, Dunbar SB, Higgins MK, et al. Combined exercise and cognitive behavioral therapy improves outcomes in patients with heart failure. J Psychosom Res 2010;69:119-31.

31. Chaytor N, Ciechanowski P, Miller JW, et al. Long-term outcomes from the PEARLS randomized trial for the treatment of depression in patients with epilepsy. Epilepsy Behav 2011;20:545-9.

32. Rickhi B, Moritz S, Reesal R, et al. A spirituality teaching program for depression: a randomized controlled trial. Int J Psychiatry Med 2011;42:315-29.

33. Renton $\mathrm{T}$, Tang $\mathrm{H}$, Ennis $\mathrm{N}$, et al. Web-based intervention programs for depression: a scoping review and evaluation. $J$ Med Internet Res 2014;16:e209.

34. Rickhi B, Moritz S, Reesal R, et al. A spirituality teaching program for depression: a randomized controlled trial. Int J Psychiatry Med 2011;42:315-29.

35. Blumenthal JA, Babyak MA, Doraiswamy PM, et al. Exercise and pharmacotherapy in the treatment of Major depressive disorder. Psychosom Med 2007;69:587-96.

36. Chung LJ, Tsai PS, Liu BY, et al. Home-based deep breathing for depression in patients with coronary heart disease: a randomised controlled trial. Int J Nurs Stud 2010;47:1346-53.

37. Ciechanowski P, Chaytor N, Miller J, et al. PEARLS depression treatment for individuals with epilepsy: a randomized controlled trial. Epilepsy Behav 2010;19:225-31.

38. Ciechanowski P, Wagner E, Schmaling K, et al. Communityintegrated home-based depression treatment in older adults: a randomized controlled trial. JAMA 2004;291:1569-77.
39. Naylor EV, Antonuccio DO, Litt M, et al. Bibliotherapy as a treatment for depression in primary care. J Clin Psychol Med Settings 2010;17:258-71.

40. Joling KJ, van Hout HP, van't Veer-Tazelaar PJ, et al. How effective is bibliotherapy for very old adults with subthreshold depression? A randomized controlled trial. Am J Geriatr Psychiatry 2011;19:256-65.

41. Higgins JP, Altman DG, Gøtzsche PC, et al. The cochrane collaboration's tool for assessing risk of bias in randomised trials. BMJ 2011;343:d5928

42. Higgins JP, Thompson SG, Deeks JJ, et al. Measuring inconsistency in meta-analyses. BMJ 2003;327:557-60.

43. Caldwell DM, Ades AE, Higgins JP. Simultaneous comparison of multiple treatments: combining direct and indirect evidence. $B M$ 2005;331:897-900

44. Cohen J. Statistical power analysis for the behavioral sciences. 2nd ed. Hillsdale: Lawrence Erlbaum, 1988.

45. Faraone SV. Interpreting estimates of treatment effects: implications for managed care. $P$ T 2008;33:700-11.

46. Cuijpers $P$, van Straten A, van Schaik A, et al. Psychological treatment of depression in primary care: a meta-analysis. $\mathrm{Br} J \mathrm{Gen}$ Pract 2009:59:51-60.

47. Krishna M, Lepping P, Jones S, et al. Systematic review and metaanalysis of group cognitive behavioural psychotherapy treatment for sub-clinical depression. Asian J Psychiatr 2015;16:7-16.

48. Wegner M, Helmich I, Machado S, et al. Effects of exercise on anxiety and depression disorders: review of meta- analyses and neurobiological mechanisms. CNS Neurol Disord Drug Targets 2014;13:1002-14.

49. Matta Mello Portugal E, Cevada T, Sobral Monteiro-Junior R, et al. Neuroscience of exercise: from neurobiology mechanisms to mental health. Neuropsychobiology 2013;68:1-14.

50. Schuch FB, Vasconcelos-Moreno MP, Borowsky C, et al. Exercise and severe major depression: effect on symptom severity and quality of life at discharge in an inpatient cohort. J Psychiatr Res 2015;61:25-32

51. Krogh J, Nordentoft M, Sterne JA, et al. The effect of exercise in clinically depressed adults: systematic review and meta-analysis of randomized controlled trials. J Clin Psychiatry 2011;72:529-38.

52. Danielsson L, Noras AM, Waern M, et al. Exercise in the treatment of major depression: a systematic review grading the quality of evidence. Physiother Theory Pract 2013;29:573-85.

53. Mura G, Moro MF, Patten SB, et al. Exercise as an add-on strategy for the treatment of major depressive disorder: a systematic review. CNS Spectr 2014;19:496-508.

54. Efthimiou O, Debray TP, van Valkenhoef G, et al. GetReal in network meta-analysis: a review of the methodology. Res Synth Methods 2016;7:236-63. 